\title{
Developing clinical reasoning in the classroom - analysis of the 4C/ID-model
}

\author{
T.C. Postma* and J.G. White
}

Department of Dental Management Sciences, School of Dentistry, University of Pretoria, Pretoria, South Africa

* Correspondence

Thomas C. Postma

Department of Dental Management Sciences

School of Dentistry

University of Pretoria

Corner of Dr Savage Rd and Bophelo Road

Gezina 0084

Pretoria

South Africa

Tel: +27 123192553 begin_of_the_skype_highlighting

$+2712$

3192553 FREE end_of_the_skype_highlighting

Fax: +27 123192171

email: corne.postma@up.ac.za

\section{Abstract}

Objective: The purpose of this paper is to provide a theoretical rationale for the implementation of the Four Component Instructional Design Model for Complex Learning (4C/ID-model) at the University of Pretoria with the aim of systematically developing the clinical reasoning of undergraduate dental students in a classroom setting.

Methods: Recent literature provides a rationale for the use of the 4C/ID-model to teach clinical reasoning in terms of four strategic teaching and learning needs: (i) the need for authenticity in the learning process; (ii) the need to manage cognitive load during the learning process; (iii) the need for repeated practise; as well as a need for (iv) valid assessment, feedback and reflection. 
Results and discussion: The literature review indicated that the 4C/ID-model makes provision for teaching and learning in an authentic context and proposes systematically structured methods that could be practically applied to manage cognitive load during repetitive exercises. The model also makes provision for cognitive feedback following assessment in order to eliminate misconceptions about content and to develop the cognitive strategies of the learner.

Conclusion: On the basis of the literature review above it is recommended that the 4C/IDmodel be considered as a basis for classroom teaching and learning to develop competence in clinical reasoning in undergraduate dental students, even at pre-clinical level. The model may also have an application in medical education.

\section{Introduction}

Clinical reasoning is an ambiguous concept $(1,2)$ consisting of interactions between the patient and the clinician within the broader social environment $(3,4)$. This haziness is further complicated by variances in clinicians' personal and specialised knowledge, experience $(1,3)$ and personal values. These personal characteristics are by default transferred to, and applied in, the so-called "clinical reasoning process" $(3,4)$. The fact of the matter is that human behaviour remains unpredictable, regardless of scientists' efforts to understand and theorise the mechanisms which are used by humans for processing information (5). Therefore, it is not surprising that no conceptual model exists that defines clinical reasoning or the clinical reasoning process unambiguously $(3,6)$ Norman (1) even goes so far as to say that clinical reasoning may not even be a valid construct and that expertise in this regard is merely the accessibility of "multiple representations of knowledge" stored as "concepts" and "conceptual knowledge" in the mind of the 
clinician (1). Despite the lack of clarity about clinical reasoning as a concept and the apparent non-existence of a distinct clinical reasoning process (1), it can be safely argued that the development of competence in clinical reasoning remains a very important aspect of the education of medical and dental students at undergraduate level $(1,2)$.

In the past it was suggested that students be taught based on the reasoning processes observed in experts. However, with the reasoning processes of experts being variable and undefined (1), instructional designers are sent back to the drawing board with the challenge to find appropriate teaching strategies to facilitate the development of clinical reasoning skills. Four strategic issues in this area of interest emerged from recent literature. These are the need for: authenticity in the learning process $(2,7)$; managing cognitive load during the learning process (7); repeated practise $(1,2)$; and valid assessment (8), feedback and reflection $(1,2)$.

In dentistry, clinical reasoning could either be "practised" clinically at the chair-side or in the classroom. Although the recent literature contains well-defined teaching strategies (9) that could be used at the chair-side, teaching and learning in the clinical area are generally incidental and variable because real-life clinical cases are inherently different from each other. Strategies to systematically develop competence in clinical reasoning at the chair-side will therefore be less than ideal because individual students will not be exposed to the same cases. This problem can be overcome by making use of standardised case studies in the classroom setting to systematically develop competence in clinical reasoning. Case-based teaching is commonly used in dental education to integrate different disciplines in dental curricula (9-11). 'Good' case studies are constructed to be realistic, relevant, challenging, engaging and educational (12). Case-based teaching and 
learning can foster critical thinking and develop decision-making skills $(13,14)$. Despite these positive perceptions about case-based teaching and learning, a relative dearth of information exists within the domain of medical and dental education regarding systematic teaching strategies that could be used to develop clinical reasoning skills in novices in a classroom setting. Masterfully designed archetypes, however, exist in the domain of cognitive psychology. One such model is the Four Component Instructional Design Model for Complex Learning (referred to from now onwards as the 4C/ID-model) (15).

The 4C/ID-model (15) propounds that the human memory is only able to handle a limited amount of information at a given point in time. If the necessary knowledge structures are not in place, exposure to a complex case will result in cognitive overload. "Cognitive overload" refers to a state where the working memory cannot handle the amount of information the learner is bombarded with at one time, which leads to a breakdown in learning processes (15). The 4C/ID-model (15) proposes strategies to systematically manage cognitive load during the teaching and learning process. The model supports the notion that repeated exposure to a complex problem in an authentic way, together with proper learner support (scaffolding), will reduce the load on the memory, which will enhance the development of integrated knowledge structures (15). The model's detailed description of a methodical instructional design framework to support the learning process and its integration of cognitive science and educational theory make the model an attractive option to the educator.

Hence, the objective of this research was to explore the potential use of the 4C/ID-model (15) as a basis for case-based classroom teaching and learning in order to develop clinical 
reasoning skills in undergraduate dental students. The purpose of this paper is to provide the theoretical background for an action research project ( $\mathrm{PhD}$ Protocol 153/2009 ${ }^{1}$ ) conducted from 2009 to 2011 at the School of Dentistry, University of Pretoria, South Africa. This paper therefore serves as a position paper for future reports on the implementation and evaluation of case-based teaching and learning strategies based on the 4C/ID-model (15) at the University of Pretoria.

\section{Methods}

This paper is written in the form of a literature review. The literature review started in 2008 as part of a needs assessment for a new curriculum to develop clinical reasoning skills in dental students at the University of Pretoria. At that stage, the 4C/ID- model (15) was the only model found in the literature that was provided a detailed design to support teaching and learning from a novice to an expert level based on cognitive science theory.

A literature search pertaining to the theoretical grounding of clinical reasoning was conducted on Eric and Medline (Proquest), targeting peer-reviewed literature (1975current)". A combination search using the keywords "clinical reasoning" and "medical education" rendered 757 results. A combination search using "clinical reasoning" and "dental education" rendered a further 116 results. The summaries and abstracts of the identified literature were screened for appropriateness. On the basis of this assessment 120 full text papers were studied. Two current review articles $(1,2)$ were identified to substantiate the current theoretical view point on the topic. Additional literature identified from quotations in the full text papers was also included in the review.

\footnotetext{
${ }^{1}$ Ethical approval was obtained from the Research Ethics Committee of the Faculty of Health Sciences, University of Pretoria for the broader action research project
} 


\section{Results and discussion}

The 4C/ID-model (15) and related educational theory regarding "clinical reasoning" were studied in order to draw a synthesis that would describe the potential application of the 4C/ID-model for classroom teaching and learning in terms of the four strategic issues identified in the introduction of this paper. The results are as follows.

\section{The need for authenticity in the learning process}

Adult learning theory suggests that learning is more likely to occur in an authentic context $(2,7)$. The 4C/ID-model propagates the use of a "whole-task" approach in order to achieve authenticity in the learning process. The "whole-task" approach is an approach that deals with a task in its entirety to ensure the integration of knowledge (15). A "whole-task" approach can therefore be defined as a task that is executed in a real life authentic situation or a simulation task where all the information is available to the student. The task referred to in this instance is the segment of a dental visit during which the dentist gathers information, makes a diagnosis and formulates a treatment plan in order to manage the patient's conditions and complaints. For the purpose of classroom teaching and learning the task will obviously be translated into a case study containing an element of all of the aforementioned aspects of the dental visit.

Introducing all of these elements into one case study, however, immediately raises the concern of complexity (15).

\section{The need to manage cognitive load during the learning process}

Innately, the application of the complex skills mentioned in the paragraphs above might be challenging to an inexperienced student. To exacerbate the natural complexity of the 
situation, a dental disease or condition rarely occurs in isolation. An adult person potentially has from nought to 32 teeth (Figure 1). Each tooth can be divided into five crown surfaces and has one or multiple roots. Even the surrounding soft tissues can be demarcated into different anatomical structures or sites. Each of these sites, or combination of sites, might be affected by dental disease in varying degrees. The completion of records as illustrated in Figure 1 is inherently a complex psychomotor exercise, and its interpretation is not easy either. Furthermore, the treatment plan decisions based on these records might depend on the psychosocial status of the patient $(4,16)$ as well as factors such as resources in the healthcare environment (17). Typically, a learner has to integrate all of the above-mentioned information to effectively manage the treatment of the patient comprehensively.

Having elaborated on the complexity of the task at hand it becomes clear that it will not always be feasible to teach all of these integrated skills simultaneously and that strategies will be required in the instructional design to manage the cognitive load $(7,15)$. As a solution to this predicament, the 4C/ID-model (15) suggests a "part-task" approach whereby the task may be broken into smaller parts to avoid cognitive overload. The disadvantage of a "part-task" approach is that inexperienced learners may not understand the broader context of the learning, which may lead to perceptions that the learning may not be relevant and a subsequent breakdown in the learning process (15). The dilemma of losing relevance can partially be overcome by making use of what is described in the 4C/ID-model as a "part-whole" approach, according to which a large number of elements of the broader context is retained in the "part-task" approach to enable the inexperienced student to realise where the learning fits into the bigger picture (15). In fact, Khatami, et al 
practically showed that dental students tend to reason using "part-whole" approaches, based on their frame of reference and previous experience (17).

It goes without saying that when one is working with inexperienced undergraduate students, teaching and learning should commence with simpler cases (2). This requirement stems from evidence that suggests that novices possess fewer knowledge structures and that these knowledge structures are less developed than those of experts (18). To manage the cognitive load during the systematic learning process, the 4C/IDmodel proposes multiple tasks sequentially ordered from easy to difficult (15). Tasks that are grouped according to level of difficulty are called "task classes" (Figure 2). There may be more than one task of more or less equal difficulty in a specific "task class". A student should ideally progress to the next "task class" when the current "task class" is mastered.

In reality, the requirement of the model to categorise cases according to level of difficulty will not be straightforward, as a result of the inherent variability of clinical cases in general. A systematic approach to solve this dilemma may be to concentrate on a single commonly encountered dental disease such as dental caries in the first "task class". Other conditions and diseases can then be systematically added to the case studies to form the subsequent "task classes". The second "task class" could, for example, contain dental caries and tooth wear, while the third "task class" could be dental caries, tooth wear and periodontal disease, and so on. Using such an approach the application of the content of the previous "task class" will be reinforced while the new condition becomes the cognitive challenge in the subsequent "task class". 
Since it is proposed in the literature that even pre-clinical students (2) be targeted, it is suggested that the initial "task classes" should focus on the most commonly found diseases and conditions. Kessner et al (19), advocated the use of so-called "tracer conditions". The use of "tracer conditions" is a sampling strategy that focuses on diseases and conditions that are commonly found. The use of such a strategy will ensure a high degree of relevance in the educational process because the strategy will be based on what most dentists are exposed to most of the time $(19,20)$. The World Health Organization (WHO) draws attention to the most important oral and dental diseases as evidenced by epidemiological studies (21). A strong argument can therefore be made that the diagnosis and management of the above-mentioned "tracer conditions" should form the content basis of the initial "task classes" to teach inexperienced students. Less common diseases and conditions may be equally important from a content point of view but it will not be feasible to cover hundreds of conditions in case study designs - as proposed above. Furthermore, an early introduction to hundreds of less frequently found conditions to a novice will surely result in cognitive overload. These conditions should therefore be systematically covered at an appropriate time elsewhere in the curriculum. Darling and Daley (22) proposed useful strategies that could be employed for the selection of pathological conditions that should be taught as part of a dental curriculum.

Having dealt with scaffolding strategies related to content selection, the focus now shifts towards the management of cognitive load during the teaching and learning process. A key principle of the 4C/ID-model (15) is that learner support should be provided at the beginning of a "task class" and should be gradually reduced in subsequent tasks before the next "task class" is proceeded to (Figure 2). The model allows for two types of support to the learner. 
The first type of support is product-orientated "supportive information" (Figure 2) (15). "Supportive information" is the bridge between existing knowledge (Figure 3) and the task at hand (15). "Supportive information" may be in the form of a lecture, textbooks, lecture notes, a model answer to a similar problem, the Internet, an answer to a question posed by the student, or feedback to the student (15). The 4C/ID-model proposes that new knowledge is constructed by providing "supportive information" in combination with the elaboration of previously mastered knowledge (Figure 3) (15). This concept is similar to the learning and development theories of Lev Vygotsky (23). Vygotsky's theories suggest that previously mastered knowledge becomes the basis for new learning (23). Vygotsky's theories suggest that further learning is most likely to occur in the socalled "zone of proximal development", which can be defined as the potential learning that could take place when the inexperienced leaner is actively supported by a more experienced teacher (23). In other words it can be argued that the knowledge gained from an earlier "task class" serves as the basis for new learning in the subsequent "task class", which could be deemed to be synonymous with the "zone of proximal development" (23). According to the 4C/ID-model new "supportive information" should be linked to specific "task classes" and should be made available as such. The 4C/ID-model makes provision for the introduction of "supportive information" by means of either inductive or deductive methods (15). Deductive methods typically may include theory followed by a case study while inquiry methods usually start with the problem followed by exploration by the student and/or through explanation from faculty. On the one hand, inquiry methods requiring independent exploration by the student inherently place a high 
burden on cognitive load and are certainly not the method of choice to teach a novice (15). On the other hand, pure deductive- or information-gathering methods may be equally unacceptable because such methods do not necessarily promote the application of prior knowledge and its synthesis with existing knowledge (15, 24, 25). Moreover, inquiry methods are likely to require more time and resources compared to deductive methods (25). A balance should therefore be struck between the selection of inquiry and deductive methods based on what is feasible in the physical teaching and learning environment (25). Regardless of the chosen method, appropriately designed and administered "supportive information" is critical to reduce the cognitive load during the learning process, especially for the inexperienced undergraduate student.

The 4C/ID-model also makes provision for a second type of learner support in the form of just-in-time process-orientated support according to which information about the learning process (Figure 2) - for example the steps used during the diagnostic process and the treatment plan formulation - is provided to the student at the exact time the student needs the information (15). In reality this could be verbal instructions from an expert (high-level support) or written instructions describing the steps of the task (low-level support). This type of support is provided to reduce the cognitive load and to allow the student to concentrate on the content aspects of the task. This type of support can generally be faded over time as the steps of the process become internalized in the mind of the student after repeated exercise (15). 


\section{The need for repeated practise}

Studies indicate that learners go through four phases to establish rich knowledge networks, which eventually constitute clinical proficiency (26). The learning process starts off with the acquisition of textbook knowledge, and this is followed by the formation of simplistic mental models (Figure 3) of diagnostic information through practise (15). Repeated experience leads to the development of so-called "illness scripts" (27) that are further elaborated on and refined and then stored (28). Illness scripts are defined as sequences of ordered information (29), such as the linked attributes of a disease (29). Accessing rich knowledge structures requires "forceful features" (31). Forceful features can be defined as distinct fragments of information - such as clinical observations or specific information received from the patient - that serve as the key to unlock memory structures that enable the interpretation of clinical information (31). A less complicated interpretation of all of these theories is that the application of relevant information through repeated practise will refine knowledge structures $(1,2)$ and access to the important "concepts" and "conceptual knowledge" $(1,15)$, which ultimately should improve clinical decision making $(2,32-36)$. The 4C/ID-model methodically provides the instructional designer with methods to reinforce learning by means of repetition as well as strategies to continuously add new knowledge to the existing knowledge base through its "task class" approach (Figure 2) (15).

The focus of the deliberations up to this point has been teaching and learning. The final section of this essay will address the outstanding issues of assessment, feedback and reflection. 


\section{The need for valid assessment, immediate feedback and reflection}

Essays with descriptions of the actual reasoning and multiple-choice questions are feasible tools to assess competence in clinical reasoning $(8,37)$. Multiple-choice questions, articulated on an appropriate knowledge-application level, in particular, have been shown to be valid and reliable for this purpose $(8,37,38)$. Both essays and multiple-choice questions can be used for formative and summative assessment purposes. Logically, assessment should be aligned (39) with teaching and learning. Assessment, in turn, ought to be followed by cognitive feedback to support the learning process (15). "Multiple short problems" may be appropriate to test the outcome of the reasoning (1) while essays could contain descriptions or syntheses of the reasoning process (8), which will be open to feedback about the students' thinking processes. Cognitive feedback (Figure 3) forms an integral part of the 4C/ID-model and is labelled as a form of "supportive information" (15). Properly designed feedback processes have the potential to motivate learners to reflect on their performance $(1,2)$. Feedback is important to eliminate misconceptions about content and to assist in the establishment and refinement of the cognitive strategies of the student $(1,2,15)$.

Cognitive strategies are defined in the 4C/ID-model as thinking processes used by the student during the reasoning process (15). Examples of two documented cognitive strategies employed during the reasoning process are hypothetico-deductive reasoning (40) and pattern recognition (41). It has been postulated that the analytical process of hypothetico-deductive reasoning is hypothesis driven and hypotheses are either accepted or rejected in the thinking process and require forward and backward reasoning (41). Pattern recognition, in contrast to hypothetico-deductive reasoning, is a non-analytical process according to which clinicians recognise diseases intuitively (2) on the basis of 
previous experience (41). The diagnosis of dental caries is often based on pattern recognition because of the typical pattern-like presentation of the signs and symptoms of the disease (42). The exclusive use of non-analytical methods, such as pattern recognition, might not be beneficial as it might result in misconceptions and misdiagnoses if alternatives are not considered (41). These negative remarks do not imply that methods such as pattern recognition are inferior to analytical approaches $(1$, 41). In fact, pattern recognition is a trademark of expert reasoning (41). Therefore, it can be argued that both analytical and non-analytical reasoning strategies should be developed. A bigger need, however, exists to develop the students' metacognition abilities to recognize and correct misconceptions during the reasoning process (2). This can only be achieved through just-in-time feedback (2) focussed on the cognitive strategies followed by the student - a principle that is strongly supported by the 4C/IDmodel (15).

\section{Conclusion}

The 4C/ID-model supports the notion of repeated exercise in an authentic context and provides substantial detail of how the teacher should manage the cognitive load of novices. The model therefore appears to be a suitable blueprint for classroom teaching and learning, even at a pre-clinical level, with the aim of developing clinical reasoning skills in undergraduate dental students, with the use of a case-based approach.

On the basis of the analysis set out above, it is recommended that the 4C/ID-model be evaluated as a basis for classroom teaching and learning to develop clinical reasoning skills in undergraduate dental students. 


\section{References}

1 Norman G. Research in clinical history: past history and current trends. Med Educ 2005: 39: 418-427.

2 Kassirer JP. Teaching clinical reasoning: Cased-based and coached. Acad Med 2010: 85: 118-124.

3 Higgs J, Jones N. Clinical decision making and multiple problem spaces. In: Higgs J, Jones MA, Loftus S, Christensen N, eds. Clinical reasoning in the health professions. Oxford, UK: Butterworth-Heinemann, 2008: 3-17.

4 Khatami S, Macentee MI. Evolution of clinical reasoning in dental education. J Dent Educ 2011: 75: 321-328.

5 Bargh JAC, Chartrand T.L. The unbearable automaticity of being. Am Psychol 1999: 54: 462-479.

6 Charlin B, Lubarsky S, Millette B, et al. Clinical reasoning processes: unravelling complexity through graphical representation. Med Educ 2012: 46: 454-463.

7 Van Merrienboer JJG, Sweller J. Cognitive load theory and complex learning: recent developments and future directions. Educ Psychol Rev 2005: 17: 147-177.

8 Norcini J, Anderson B, Bollela V, et al. Criteria for good assessment: consensus statement and recommendations from the Ottawa 2010 Conference. Med Teach. 2011: 33: 206-214.

9 McMillan WJ. Teaching for clinical reasoning - helping students make the conceptual links. Med Teach 2010: 32: 436-442.

10 Garvey MT, O'Sullivan M, Blake M. Multidisciplinary case-based learning for undergraduate students. Eur J Dent Educ 2000: 4: 165-168.

11 Engel FE, Hendricson WD. A case-based learning model in orthodontics. J Dent Educ 1994: 58: 762-767. 
12 Kim S, Phillips WR, Pinsky L, Brock D, Phillips K, Keary J. A conceptual framework for developing teaching cases: a review and synthesis of the literature across disciplines. Med Educ 2006: 40: 867-876.

13 Boehrer J, Linsky M. Teaching with cases: learning to question. New Dir Teach Learn 1990: 42: 41-57.

14 Richards PS, Inglehart MR. An interdisciplinary approach to case-based teaching: does it create patient-centered and culturally sensitive providers? J Dent Educ 2006: 70: 284-291.

15 Van Merriënboer JJ, Clark RE, De Croock MB. Blueprints for complex learning: the 4C/ID-model. Education Tech Research and Dev. 2002: 50: 39-64.

16 Suls J, Rothman A. Evolution of the biopsychosocial model: prospects and challenges for health psychology. Health Psychol 2004: 23: 119-125.

17 Khatami S, Macentee MI, Pratt DD, Collins JB. Clinical reasoning in dentistry: A conceptual framework for dental education. J Dent Educ 2011: 76: 1116-1128.

18 Bordage G, Lemieux M. Semantic structures and diagnostic thinking of experts and novices. Acad Med 1991: 66: $\operatorname{suppl(9):~s70-72.~}$

19 Kessner DM, Kalk CE, Singer J. Assessing health quality - the case for tracers. N Engl J Med: 1973: 288: 189-194.

20 Norcini JJ. Current perspectives in assessment: the assessment of performance at work. Med Educ 2005: 39: 880-889.

21 Petersen PE, Bourgeois D, OgawaI H, Estupinan-Day S, Ndiaye C. The global burden of oral diseases and risks to oral health. Bull World Health Organ 2005: 83: 661-669.

22 Darling MR, Daley TD. Oral pathology in the dental curriculum: A guide on what to teach. J Dent Educ 2006: 70: 355-360. 
23 Harland T. Vygotsky's Zone of Proximal Development and Problem-based Learning: Linking a theoretical concept with practice through action research. Teaching in Higher Education. 2003. 8: 263-272.

24 Harden RM, Sowden S, Dunn WR. Educational strategies in curriculum development: the SPICES model. Med Educ 1984: 18: 284-297.

25 Harden RM, Davis MH. The continuum of problem-based learning. Med Teach. 1998: 20: 317-322.

26 Schmidt HG, Norman GR, Boshuizen HP. A cognitive perspective on medical expertise: theory and implication. Acad Med 1990: 65: 611-621.

27 Johnson MK, Hasher L. Human learning and memory. Annu Rev Psychol 1987: 38: $631-668$.

28 Schmidt HG, Norman GR, Boshuizen HP. A cognitive perspective on medical expertise: theory and implication. Acad Med 1990: 65: 611-621.

29 Fayol M, Monteil JM. The notion of script: from general to developmental and social psychology. Curr Psychol Cogn. 1988: 8: 335-361.

30 Charlin B, Tardif J, Boshuizen HP. Scripts and medical diagnostic knowledge: theory and applications for clinical reasoning instruction and research. Acad Med 2000: 75: 182-190.

31 Grant J, Marsden P. The structure of memorized knowledge in students and clinicians: an explanation for diagnostic expertise. Med Educ 1987: 21: 92-98.

32 Moulaert V, Verwijnen MG, Rikers R, Scherpbier AJ. The effects of deliberate practice in undergraduate medical education. Med Educ 2004: 38: 1044-1052.

33 Rikers RM, Loyens SM, Schmidt HG. The role of encapsulated knowledge in clinical case representations of medical students and family doctors. Med Educ 2004: 38: 1035-1043. 
34 Woods NN, Brooks LR, Norman GR. The value of basic science in clinical diagnosis: creating coherence among signs and symptoms. Med Educ 2005: 39: $107-112$.

35 Woods NN, Howey EH, Brooks LR, Norman GR. Speed kills? Speed, accuracy, encapsulations and causal understanding. Med Educ 2006: 40: 973-979.

36 Ericsson KA. Deliberate practice and the acquisition and maintenance of expert performance in medicine and related domains. Acad Med 2004: 79: suppl(10): s70-81.

37 Wass V, Van der Vleuten C, Shatzer J, Jones R. Assessment of clinical competence. Lancet 2001: 357: 945-949.

38 Palmer EJ, Devitt PG. Assessment of higher order cognitive skills in undergraduate education: modified essay or multiple choice questions? Research Paper. BMC Medical Education 2007: 7:49 doi:10.1186/1472-6920-7-49

39 Biggs J. Enhancing teaching through constructive alignment. Higher Educ 1996: 32: $347-364$.

40 Barrows HS, Norman GR, Neufeld VR, Feightner JW. The clinical reasoning of randomly selected physicians in general medical practice. Clin Invest Med 1982: 5: 49-55.

41 Eva KW. What every teacher needs to know about clinical reasoning. Med Educ 2004: 39: 98-106.

42 Bader JD, Shugars DA. What do we know about how dentists make caries-related treatment decisions? Community Dent Oral Epidemiol 1997: 25: 97-103. 


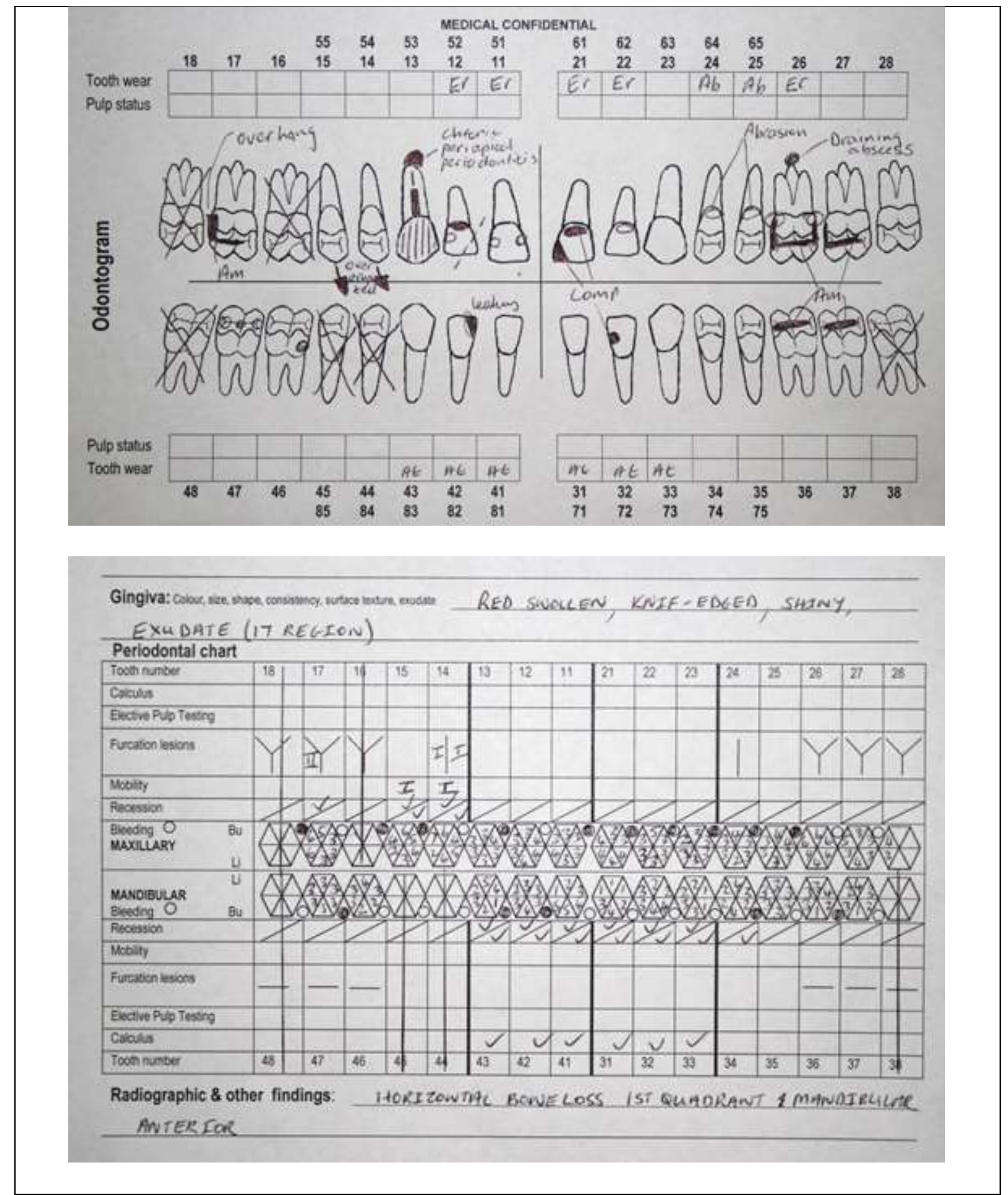

Fig. 1. A typical dental record displaying complex disease patterns 


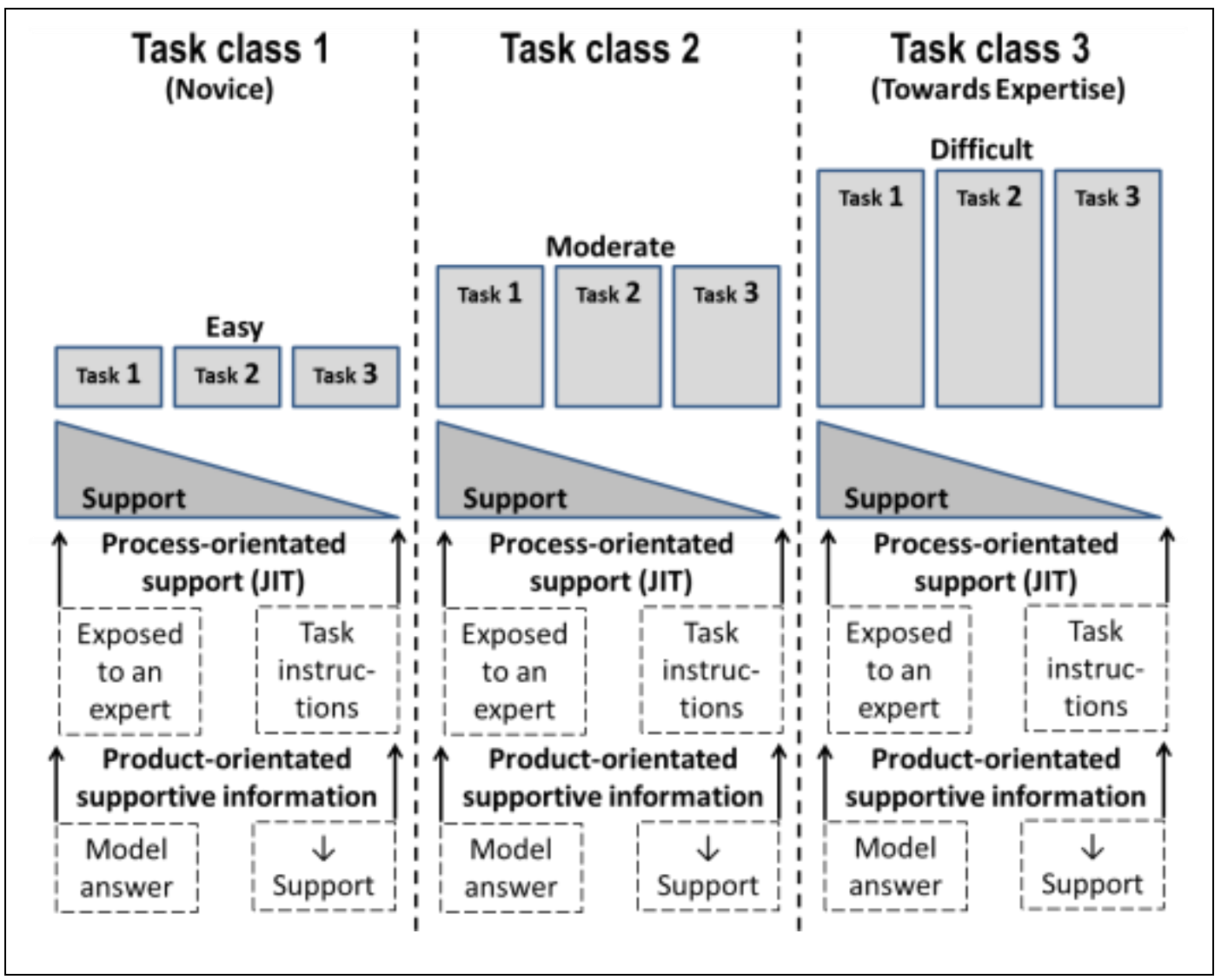

Fig. 2. The task-class concept with diminishing learner support ${ }^{*}$

*Adapted from the Four Component Instructional Model for Complex Learning ${ }^{15}$ 


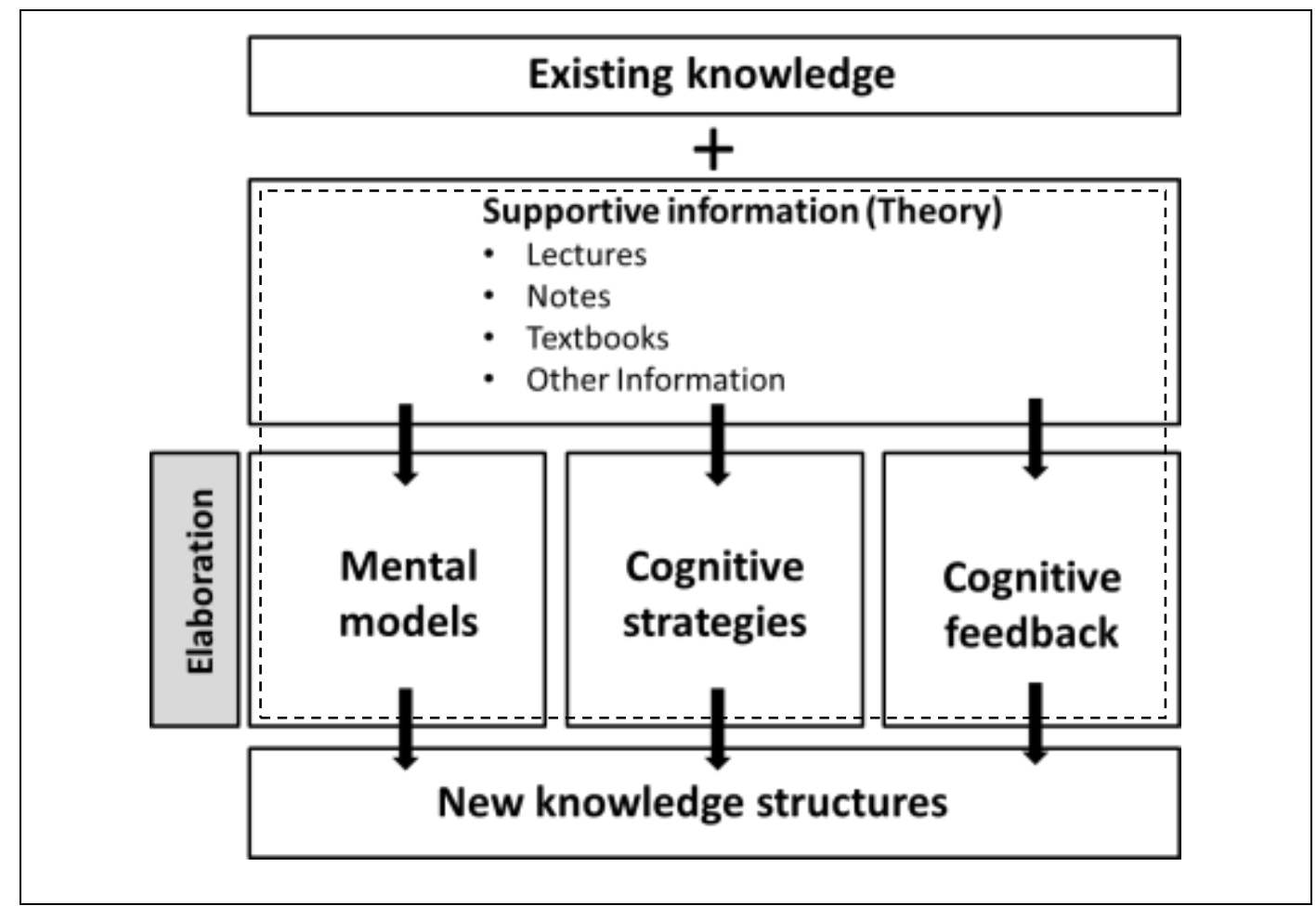

Fig. 3. Supportive information - the bridge between existing and new knowledge*

*Adapted from the Four Component Instructional Model for Complex Learning ${ }^{15}$ 